\title{
A Successive Intercarrier Interference Reduction Algorithm for OFDM Systems
}

\author{
Y. H. Zhang, W.-S. Lu, and T. A. Gulliver \\ Department of Electrical and Computer Engineering, University of Victoria \\ P.O. Box 3055, Victoria, B.C., Canada V8W 3P6 \\ Email: yhzhang, wslu, agullive@ece.uvic.ca
}

\begin{abstract}
In a rapidly fading environment, Doppler spread caused by user mobility destroys the orthogonality among OFDM subcarriers, resulting in intercarrier interference. In this paper, a low complexity ICI reduction algorithm which can be applied to QAM signal constellations is proposed. A combinatorial optimization problem for ICI suppression is formulated and then relaxed to a quadratic programming problem. A successive method is then utilized to deduce a sequence of reduced-size QP problems, which is solved by limiting the search in the 2dimensional subspace spanned by its steepest-descent and Newton directions to reduce the computational complexity. Furthermore, a low-bit descent search is employed to enhance the system performance. The proposed algorithm is shown to provide excellent performance with low computational complexity.
\end{abstract}

\section{INTRODUCTION}

Orthogonal frequency division multiplexing (OFDM) modulation has been widely used in communication systems to meet the demand for ever increasing data rates. It is robust over multipath fading channels and results in significantly reduced receiver complexity [1]. The standards employing OFDM modulation include digital video broadcasting (DVB) [2], digital audio broadcasting DAB [3], IEEE 802.11a and $802.11 \mathrm{~g}$ [4] for wireless local area networks, and IEEE 802.16 [5] for wireless metropolitan area networks.

In an OFDM system, the data stream is divided into $N$ parallel lower-rate data streams, which are modulated onto $N$ orthogonal subcarriers. In a rapidly fading environment, the channel can be time varying even within one symbol duration. Doppler spread caused by user mobility then destroys the orthogonality among subcarriers, which results in intercarrier interference (ICI) and degraded system performance [6], [8].

A number of algorithms have been proposed to mitigate the ICI and improve system performance over doubly-selective channels. In [6], Li and Cimini provide universal bounds on the ICI in an OFDM system over time-varying fading channels, which can be evaluated and compared with the exact ICI. An ICI suppression algorithm using parallel canceling with frequency-domain equalization techniques is presented in [7], where two-stage prefilters and ICI reduction filters are utilized to achieve minimum mean square error (MMSE) equalization. However, it is assumed that the channel varies linearly during one symbol duration. In [8], a block decision feedback equalizer (DFE) algorithm is described, which only utilizes signals from several neighboring subcarriers to eliminate the ICI for a certain subcarrier. Kou et, al. [9] proposed a low complexity ICI reduction algorithm based on an iterative optimization scheme, but it is appropriate only for OFDM systems with 4 quadrature amplitude modulation (4-QAM). In [8] and [9], by exploiting the frequency diversity introduced by channel variations, better performance was obtained for channels with larger Doppler spread. Furthermore, Stamoulis et al. [10] derived linear time-varying filters in MIMO OFDM systems that maximize the ratio of signal energy to ICI-plusnoise energy.

In this paper, a low complexity ICI reduction algorithm is proposed which is applicable to QAM signal constellations for OFDM systems with doubly-selective fading channels. A combinatorial optimization problem for ICI suppression is formulated, and then relaxed to a quadratic programming problem. A successive method is then utilized to deduce a sequence of reduced-size QP problems, which is solved by limiting the search in the 2-dimensional subspace spanned by its steepestdescent and Newton directions to reduce the computational complexity. Furthermore, a low-bit descent search (LBDS) is employed to improve the system performance. Performance results are given to demonstrate that the proposed algorithm provides excellent performance with low computational complexity.

\section{SYSTEM MODEL}

In an OFDM system, the system bandwidth is divided into $N$ subchannels, and the data stream is modulated on the subcarriers using QAM or phase-shift keying (PSK). The transmitted signal is generated using an inverse fast Fourier transform (IFFT)

$$
x_{n}=\frac{1}{\sqrt{N}} \sum_{k=0}^{N-1} X_{k} \exp \left(\frac{j 2 \pi k n}{N}\right) \text { for } n=0, \ldots N-1
$$

where $x_{n}$ is the time-domain signal at the $n$th sampling instant, and $X_{k}$ is the frequency-domain data symbol for the $k$ th subcarrier. Equation (1) can be written in vector form as

$$
\mathbf{x}=\mathbf{F X}
$$

where $\mathbf{x}=\left[\begin{array}{llll}x_{0} & x_{1} & \ldots & x_{N-1}\end{array}\right]^{T}$ and $\mathbf{X}=\left[\begin{array}{llll}X_{0} & X_{1} & \ldots & X_{N-1}\end{array}\right]^{T}$ represent the time-domain and frequency-domain OFDM symbols, respectively, and $\mathbf{F}$ is the IFFT matrix with elements 
$f_{n, k}=\frac{1}{\sqrt{N}} \exp \left(\frac{j 2 \pi k n}{N}\right)$. The OFDM symbol duration is denoted by $T_{s}$, so the chip duration of each subchannel is $T_{c}=T_{s} / N$.

In this paper, we adopt a doubly frequency selective fading channel model [8], [10]. Thus, we have a wide sense stationary uncorrelated scattering (WSSUS) channel with impulse response given by

$$
h(t ; \tau)=\sum_{d=1}^{D} h\left(t ; \tau_{d}\right) \delta\left(\tau-\tau_{d}\right)
$$

where $\tau_{d}$ is the $d$ th path delay with $\tau_{1}<\tau_{2}<\ldots<\tau_{D}$. In a rich scattering environment, the channel autocorrelation function is separable in terms of time and delay [8], i.e., $\phi_{h}(\triangle t ; \tau)=\phi_{t}(\triangle t) \phi_{\tau}(\tau)$, where $\phi_{t}(\triangle t)$ is the timecorrelation function based on Jakes' model, and $\phi_{\tau}(\tau)$ is the multipath intensity profile. In $(3), h\left(t ; \tau_{d}\right)$ is a complex Gaussian process with zero mean and variance $\sigma_{d}^{2} \triangleq \phi_{\tau}\left(\tau_{d}\right)$.

A discrete version of the WSSUS channel in (3) can be modeled as a tapped delay line (TDL) with random taps [11]

$$
h(n ; l)=\sum_{d=1}^{D} h\left(n T_{c} ; \tau_{d}\right) \operatorname{sinc}\left(\frac{\tau_{d}}{T_{c}}-l\right)
$$

where $h(n ; l)$ denotes the channel coefficient for the $l$ th tap at the $n$th sampling instant, $n=0, \ldots, N-1, l=0, \ldots, L-1$ with $L=\left\lfloor\tau_{D} / T_{c}\right\rfloor+1$, and the delay between two taps is $T_{c}$.

We assume that a cyclic prefix (CP) is inserted at the beginning of each OFDM symbol before transmission, and the length $N_{p}$ of the cyclic prefix is greater than or equal to that of the channel impulse response to eliminate the intersymbol interference. Thus, the discrete signal at the receiver can be expressed as

$$
y_{n}=\sum_{l=0}^{L-1} h(n, l) x(n-l)+w_{n} \text { for } n=-N_{p}, \ldots N-1
$$

where $w_{n}$ is additive white Gaussian noise (AWGN) with zero mean and variance $\sigma^{2}$. In vector form, (5) can be written as

$$
\mathbf{y}=\mathbf{H x}+\mathbf{w}
$$

where $\mathbf{y}$ and $\mathbf{w}$ denote the time-domain received signal and AWGN noise, respectively, and $\mathbf{H}$ is the channel matrix given by

$$
\mathbf{H}=\left[\begin{array}{cccc}
h(0,0) & 0 & \ldots & h(0,1) \\
h(1,1) & h(1,0) & \ldots & h(1,2) \\
\vdots & \vdots & \ddots & \vdots \\
h(L-1, L-1) & h(L-1, L-2) & \ldots & 0 \\
\vdots & \vdots & \ddots & \vdots \\
0 & 0 & \ldots & h(N-1,0)
\end{array}\right]
$$

After removing the $\mathrm{CP}$ and performing a fast Fourier transform (FFT), we obtain

$$
\mathbf{Y}=\mathbf{A X}+\mathbf{W}
$$

where $\mathbf{Y}=\left[\begin{array}{lll}Y_{0} & \ldots & Y_{N-1}\end{array}\right]^{T}$ is the frequency-domain received signal, $\mathbf{A}=\mathbf{F}^{H} \mathbf{H F}, \mathbf{W}=\mathbf{F}^{H} \mathbf{w}$, and $H$ denotes Hermitian transpose.
If $h\left(t ; \tau_{d}\right)$ in (3) remains constant within one OFDM symbol duration, then matrix $\mathbf{A}$ is a diagonal matrix, and no ICI will occur. Conversely, if the channel varies within one OFDM symbol, the orthogonality of the subcarriers does not hold, and the received signal contains both the transmitted signal and ICI from other subcarriers [12]. In this case, the received signal on the $k$ th subcarrier is

$$
Y_{k}=A_{k, k} X_{k}+\sum_{m=0, m \neq k}^{N-1} A_{k, m} X_{m}+W_{k}
$$

where $k=0, \ldots, N-1, A_{k, m}$ denotes the $(k, m)$ th element of $\mathbf{A}$ and $\sum_{m=0, m \neq k}^{N-1} A_{k, m} X_{m}$ represents the ICI caused by other subcarriers.

\section{PROBLEM Formulation AND RELAXATION}

Based on the maximum likelihood (ML) detection criterion, the ICI reduction problem can be formulated as the optimization problem

$$
\begin{aligned}
& \operatorname{minimize}\|\mathbf{Y}-\mathbf{A X}\|_{2}^{2} \\
& \text { subject to: } X_{k} \in \mathcal{M}, \text { for } k=0,1, \ldots, N-1
\end{aligned}
$$

where $\mathcal{M}$ contains the constellation points according to the modulation being used. Since $\mathcal{M}$ is a discrete set, this is a combinatorial problem with exponential computational complexity that becomes prohibitive even for a moderate number of variables. As the first step of our solution, we propose a relaxation of the problem in (9). This relaxation allows us to consider a non-combinatorial problem that admits a fast solution which yields good performance.

The variables in (9) are complex-valued. If we define $\mathbf{Y}=\mathbf{Y}_{\mathbf{r}}+\mathbf{j} \mathbf{Y}_{\mathbf{i}}, \mathbf{A}=\mathbf{A}_{\mathbf{r}}+\mathbf{j} \mathbf{A}_{\mathbf{i}}$, and $\mathbf{X}=\mathbf{X}_{\mathbf{r}}+\mathbf{j} \mathbf{X}_{\mathbf{i}}$, then (9) becomes an optimization problem with real-valued data as

$$
\begin{aligned}
\operatorname{minimize} & \|\hat{\mathbf{Y}}-\hat{\mathbf{A}} \hat{\mathbf{X}}\|_{2}^{2} \\
\text { subject to: } & \hat{X}_{k} \in \hat{\mathcal{M}}, \text { for } k=0,1, \ldots, N-1
\end{aligned}
$$

where

$$
\hat{\mathbf{Y}}=\left[\begin{array}{l}
\mathbf{Y}_{\mathbf{r}} \\
\mathbf{Y}_{\mathbf{i}}
\end{array}\right], \hat{\mathbf{X}}=\left[\begin{array}{l}
\mathbf{X}_{\mathbf{r}} \\
\mathbf{X}_{\mathbf{i}}
\end{array}\right] \text {, and } \hat{\mathbf{A}}=\left[\begin{array}{cc}
\mathbf{A}_{\mathbf{r}} & -\mathbf{A}_{\mathbf{i}} \\
\mathbf{A}_{\mathbf{i}} & \mathbf{A}_{\mathbf{r}}
\end{array}\right] \text {. }
$$

In what follows, the OFDM system is assumed to employ 16-QAM modulation, which corresponds to $\hat{\mathcal{M}}=\{ \pm 1, \pm 3\}$. Obviously, (10) is a quadratic optimization problem with discrete variables and can be expressed as

$$
\operatorname{minimize} \hat{\mathbf{X}}^{T} \hat{\mathbf{Q}} \hat{\mathbf{X}}+\hat{\mathbf{q}}^{T} \hat{\mathbf{X}}
$$

subject to: $\hat{X}_{k}=\{ \pm 1, \pm 3\}$, for $k=0, \ldots, 2 N-1$

where $\hat{\mathbf{Q}}=\hat{\mathbf{A}}^{T} \hat{\mathbf{A}}$, and $\hat{\mathbf{q}}=-2 \hat{\mathbf{A}}^{T} \hat{\mathbf{Y}}$. The variable set in (11b) can be characterized as

$$
\hat{\mathbf{X}}=2 \boldsymbol{\alpha}+\boldsymbol{\beta}
$$


where $\boldsymbol{\alpha}$ and $\boldsymbol{\beta}$ are $2 N$-dimensional vectors with components $\alpha_{k}, \beta_{k} \in\{-1,1\}$, for $k=0, \ldots, 2 N-1$. Consequently, problem (11) assumes the form

$$
\begin{gathered}
\operatorname{minimize} \mathbf{z}^{T} \mathbf{Q z}+\mathbf{q}^{T} \mathbf{z} \\
\text { subject to: } z_{k}=\{-1,1\}, \text { for } k=0, \ldots, 4 N-1
\end{gathered}
$$

where $\mathbf{z}=\left[\begin{array}{l}\boldsymbol{\alpha} \\ \boldsymbol{\beta}\end{array}\right], \mathbf{Q}=\left[\begin{array}{cc}4 \hat{\mathbf{Q}} & 2 \hat{\mathbf{Q}} \\ 2 \hat{\mathbf{Q}} & \hat{\mathbf{Q}}\end{array}\right]$, and $\mathbf{q}=\left[\begin{array}{c}2 \hat{\mathbf{q}} \\ \hat{\mathbf{q}}\end{array}\right]$.

By realizing that the constraints in (13b) imply $\mathbf{z}^{T} \mathbf{z}=4 N$, problem (13) can be relaxed to

$$
\begin{aligned}
& \operatorname{minimize} \mathbf{z}^{T} \mathbf{Q z}+\mathbf{q}^{T} \mathbf{z} \\
& \text { subject to: } \mathbf{z}^{T} \mathbf{z}=4 N
\end{aligned}
$$

Note that (14) is an optimization problem with continuous variables. It follows from the definitions that $\hat{\mathbf{Q}}$ is a positive definite matrix and $\mathbf{Q}$ is a positive semidefinite matrix. Because of the non-convex constraint in (14b), however, (14) is not a convex QP problem. Nevertheless, an efficient solution method can be developed for problem (14). The details of this solution are given in the next section.

\section{A Successive ICI Reduction Algorithm}

\section{A. Basic Algorithm}

This section presents an ICI reduction algorithm based on the QP formulation (14). The proposed algorithm is recursive in nature, in which only some binary components of $\mathbf{z}$ in (13) are determined in each iteration by solving a corresponding non-combinatorial problem of type (14). Algorithmic details of a given, say the $i$ th, iteration are described as follows. Suppose that prior to the $i$ th iteration several binary components of vector $\mathbf{z}$ have already been determined. Let $\mathbf{z}_{i}$ be the reducedsize vector that collects all undecided components of $\mathbf{z}, \Omega_{i}$ be the index set corresponding to $\mathbf{z}_{i}$, and $N_{i}$ be the size of $\mathbf{z}_{i}$. By substituting the known binary components of $\mathbf{z}$ into (14), a reduced-size problem similar to (14) is obtained as

$$
\begin{aligned}
& \operatorname{minimize} \mathbf{z}_{i}^{T} \mathbf{Q}_{i} \mathbf{z}_{i}+\mathbf{q}_{i}^{T} \mathbf{z}_{i} \\
& \text { subject to: } \mathbf{z}_{i}^{T} \mathbf{z}_{i}=4 N_{i}
\end{aligned}
$$

The problem in (15) is solved by limiting the search in the 2dimensional subspace spanned by its steepest-descent direction (i.e., negative gradient of the objective function) and Newton direction. In doing so, we set

$$
\mathbf{z}_{i}=\eta_{1}^{(i)} \mathbf{v}_{1}^{(i)}+\eta_{2}^{(i)} \mathbf{v}_{2}^{(i)}
$$

where $\mathbf{v}_{1}^{(i)}=\mathbf{q}_{i}, \mathbf{v}_{2}^{(i)}=\mathbf{Q}_{i}^{-1} \mathbf{q}_{i}$, and $\eta_{1}^{(i)}, \eta_{2}^{(i)}$ are two scalar variables. Then (15) is reduced to the 2-dimensional problem

$$
\begin{array}{r}
\text { minimize } \boldsymbol{\eta}_{i}^{T} \boldsymbol{S}_{i} \boldsymbol{\eta}_{i}+\mathbf{p}_{i}^{T} \boldsymbol{\eta}_{i} \\
\text { subject to : } \boldsymbol{\eta}_{i}^{T} \mathbf{R}_{i} \boldsymbol{\eta}_{i}=4 N_{i}
\end{array}
$$

where $\boldsymbol{\eta}_{i}=\left[\eta_{1}^{(i)} \eta_{2}^{(i)}\right]^{T}, \mathbf{S}_{i}=\mathbf{V}_{i}^{T} \mathbf{Q}_{i} \mathbf{V}_{i}, \mathbf{p}_{i}=\mathbf{V}_{i}^{T} \mathbf{q}_{i}, \mathbf{R}_{i}=$ $\mathbf{V}_{i}^{T} \mathbf{V}_{i}$, and $\mathbf{V}_{i}=\left[\mathbf{v}_{1}^{(i)} \mathbf{v}_{2}^{(i)}\right]$. It follows from the KarushKuhn-Tucker (KKT) conditions of (17) that the solution of
(17) satisfies

$$
\begin{array}{r}
2 \mathbf{S}_{i} \boldsymbol{\eta}_{i}+\mathbf{p}_{i}+2 \lambda_{i} \mathbf{R}_{i} \boldsymbol{\eta}_{i}=0 \\
\boldsymbol{\eta}_{i}^{T} \mathbf{R}_{i} \boldsymbol{\eta}_{i}=4 N_{i}
\end{array}
$$

where $\lambda_{i}$ is a Lagrange multiplier. From (18a), the optimal $\boldsymbol{\eta}_{i}$ is given by

$$
\boldsymbol{\eta}_{i}^{*}=-\frac{1}{2}\left(\mathbf{S}_{i}+\lambda_{i}^{*} \mathbf{R}_{i}\right)^{-1} \mathbf{p}_{i}
$$

where using (18b), $\lambda_{i}^{*}$ is determined as the solution of the one-variable algebraic equation

$$
g\left(\lambda_{i}\right)=\sum_{k=0}^{N_{i}-1} \frac{\hat{p}_{k}^{2}}{\left(\lambda_{i}+s_{k}\right)^{2}}=16 N_{i}
$$

where $s_{k}$ is the $k$ th eigenvalue of $\hat{\mathbf{S}}_{i}=\mathbf{R}_{i}^{-\frac{1}{2}} \mathbf{S}_{i} \mathbf{R}_{i}^{-\frac{1}{2}}$, which admits an eigen-decomposition $\hat{\mathbf{S}}_{i}=\mathbf{U}_{i} \boldsymbol{\Sigma}_{i} \mathbf{U}_{i}^{T}$, and $\hat{p}_{k}$ is the $k$ th component of vector $\hat{\mathbf{p}}_{i}=\mathbf{U}_{i}^{T} \mathbf{R}_{i}^{-\frac{1}{2}} \mathbf{p}_{i}$. Since $g\left(\lambda_{i}\right)$ in (20) is monotonically decreasing with $\lambda_{i}$, and $g\left(\lambda_{i}\right)-16 N_{i}$ changes its sign on the interval $\left(-s_{l}, \frac{\left\|\hat{\mathbf{p}}_{i}\right\|}{1.5 \sqrt{4 N_{i}}}-s_{l}\right)$ with $s_{l}$ being the smallest value of $s_{k}$ such that $\hat{p}_{l} \neq 0$, the unique solution $\lambda_{i}^{*}$ of (20) can be effectively identified by a bisection search method. Using (16), the solution of (15) can be obtained as

$$
\mathbf{z}_{i}^{*}=\eta_{1}^{(i)^{*}} \mathbf{v}_{1}^{(i)}+\eta_{2}^{(i)^{*}} \mathbf{v}_{2}^{(i)}
$$

Next, the magnitudes of the components of $\mathbf{z}_{i}^{*}$ are examined. If $\left|z_{k}^{*}\right|$ exceeds a given threshold $\rho$, the corresponding variable is detected as $\operatorname{sign}\left(z_{k}^{*}\right)$, otherwise component $z_{k}^{*}$ remains undetermined and will be considered as a design variable in the next iteration. The components just detected are then used in (15) to produce a similar QP problem of reduced size where the vector $\mathbf{z}_{i}$ contains only the undecided variables. This iterative process continues until all the variables have been identified to produce an estimate of the transmitted data. A description of the proposed algorithm is given below:

Inputs of $i$ th iteration for $\mathbf{z}_{i}$ : The number of undecided variables $N_{i}$, the index set $\Omega_{i}$, and a given threshold $\rho$.

Step 1: Use (15) and (16) to form $\mathbf{Q}_{i}, \mathbf{q}_{i}, \mathbf{v}_{1}^{(i)}$ and $\mathbf{v}_{2}^{(i)}$.

Step 2: Generate data sets $\mathbf{S}_{i}, \mathbf{R}_{i}$ and $\mathbf{p}_{i}$; Use (19)-(21) to compute $\mathbf{z}_{i}^{*}$.

Step 3: Use the threshold $\rho$ to detect some of the binary components in $\mathbf{z}_{i}^{*}$.

Step 4: If no components of $\mathbf{z}^{*}$ are undecided, stop and output $\mathbf{z}^{*}$ as the solution vector. Compute $\hat{\mathbf{X}}^{*}$ using (2). Otherwise, update the index set to $\Omega_{i+1}$, set $i:=$ $i+1$, and repeat from Step 1 .

We conclude this section with a remark to stress that the proposed algorithm is essentially a successive two-variable optimization process, thus it is considerably more efficient than the algorithm in [9]. 


\section{B. Two Implementation Issues}

There are two issues in constructing vector $\mathbf{v}_{2}^{(i)}=\mathbf{Q}_{i}^{-1} \mathbf{q}_{i}$. The first is the existence of $\mathbf{Q}_{i}^{-1}$. At least in the first iteration where $\mathbf{Q}_{i}$ is the entire matrix $\mathbf{Q}, \mathbf{Q}^{-1}$ does not exist since $\mathbf{Q}$ is merely positive semidefinite (see (13)). This problem can be readily fixed by adding $\epsilon \mathbf{I}$ with a small $\epsilon>0$ to $\mathbf{Q}$ so that the slightly modified $\mathbf{Q}+\epsilon \mathbf{I}$ becomes positive definite, and thus nonsingular. Note that this modification does not affect the solution because the modification amounts to changing the objective function in (15a) to $\mathbf{z}_{i}^{T}\left(\mathbf{Q}_{i}+\epsilon \mathbf{I}\right) \mathbf{z}_{i}+\mathbf{q}_{i}^{T} \mathbf{z}_{i}$, which in conjunction with the constraint in (15b) equals $\mathbf{z}_{i}^{T} \mathbf{Q}_{i} \mathbf{z}_{i}+$ $\mathbf{q}_{i}^{T} \mathbf{z}_{i}+4 N_{i} \epsilon$, and adding a constant to the objective function does not alter the solution. As the iterations continue, matrix $\mathbf{Q}_{i}$ may or may not be singular, and the technique outlined above can be used in case $\mathbf{Q}_{i}$ is singular. The second issue is the evaluation of $\mathbf{Q}_{i}^{-1}$, which is numerically intensive when its size is large. This problem can be fixed using the well-known formula for inverting a four-block matrix [13], as given below.

Suppose the inverse of matrix $\mathbf{Q}_{i-1}$ is known. Since $\mathbf{Q}_{i}$ is a principal submatrix of $\mathbf{Q}_{i-1}$, simple row-and-column permutations of $\mathbf{Q}_{i}$ gives

$$
\mathbf{P Q}_{i-1} \mathbf{P}=\left[\begin{array}{cc}
\mathbf{Q}_{i} & \mathbf{B} \\
\mathbf{B}^{T} & \mathbf{C}
\end{array}\right] .
$$

Applying the formula for inverting a four-block matrix to (22), we can write

$\mathbf{P Q}_{i-1}^{-1} \mathbf{P}=\left[\begin{array}{cc}\mathbf{Q}_{i}^{-1}+\mathbf{Q}_{i}^{-1} \mathbf{B} \tilde{\mathbf{C}}^{-1} \mathbf{B}^{T} \mathbf{Q}_{i}^{-1} & -\mathbf{Q}_{i}^{-1} \mathbf{B} \tilde{\mathbf{C}}^{-1} \\ -\tilde{\mathbf{C}}^{-1} \mathbf{B}^{T} \mathbf{Q}_{i}^{-1} & \tilde{\mathbf{C}}^{-1}\end{array}\right]$

Now partition $\mathbf{P Q}_{i-1}^{-1} \mathbf{P}$, which is obtained by applying rowand-column permutations to $\mathbf{Q}_{i-1}$, into four blocks with sizes consistent with the right-hand side of (23), i.e.,

$$
\mathbf{P Q}_{i-1}^{-1} \mathbf{P}=\left[\begin{array}{cc}
\mathbf{D}_{1} & \mathbf{D}_{2} \\
\mathbf{D}_{2}^{T} & \mathbf{D}_{3}
\end{array}\right] .
$$

Then (23) and (24) imply that

$$
\mathbf{Q}_{i}^{-1}=\mathbf{D}_{1}-\mathbf{D}_{2} \mathbf{D}_{3}^{-1} \mathbf{D}_{2}^{T}
$$

where the size of matrix $\mathbf{D}_{3}$ is $N_{i-1}-N_{i}$. Since the number of variables determined in each iteration is usually small, computing $\mathbf{D}_{3}^{-1}$ is considerably more economical than computing $\mathbf{Q}_{i}^{-1}$ directly.

\section{Performance Enhancement by Low-Bit Descent Search}

In LBDS, a given binary sequence is associated with an objective function to be minimized. The search process evaluates, compares, and determines the optimal sign switches of a relatively small number of sequence components to yield maximum reduction in the objective function in (13). LBDS has been applied recently to various problems [14]. As will be demonstrated by simulation, the performance of the proposed algorithm can be considerably enhanced using 1-bit or 2-bit, or a combined 1-bit-and-2-bit LBDS, at an insignificant extra cost in computational complexity.
It turns out [14] that one-bit descent search can be carried out by evaluating $\mathbf{z} \odot \boldsymbol{\xi}$ (here $\odot$ denotes component-wise multiplication), where $\boldsymbol{\xi}=\tilde{\mathbf{Q}} \mathbf{z}+\mathbf{q} / 2$, and $\tilde{\mathbf{Q}}$ is generated from matrix $\mathbf{Q}$ with its diagonal components set to zero. Index $k^{*}$ is then identified as where the corresponding component $\xi_{k^{*}}$ has the maximum value, and the sign of $z_{k^{*}}$ is switched to obtain an improved solution. Similarly, a 2-bit LBDS is performed by computing matrix $\mathbf{G}=\boldsymbol{\xi} \boldsymbol{e}^{T}+\mathbf{e}^{T} \boldsymbol{\xi}-2 \mathbf{Q} \odot\left(\mathbf{z z}^{T}\right)$, where $\mathbf{e}$ is the all-one vector. The index $\left(k^{*}, m^{*}\right)$ is identified as where the component $P_{k^{*}, m^{*}}$ reaches maximum value, and an improved solution is then obtained by switching the signs of the $k^{*}$ th and $m^{*}$ th components of $\mathbf{z}^{*}$.

\section{Computational Complexity}

For the sake of simplicity, only multiplications are considered here. The basic algorithm involves computing (i) the inverse of the initial matrix $\mathbf{Q}$ in (14); (ii) the inverse of the reduced size matrices $\mathbf{Q}_{i}$ for $i=1,2, \ldots, K$ where $K$ denotes the number of iterations performed to complete the detection process; (iii) the data set $\left\{\mathbf{S}_{i}, \mathbf{R}_{i}, \mathbf{p}_{i}\right\}$ for $i=1,2, \ldots, K$; and (iv) the solution of the problem in (17) for $i=1,2, \ldots, K$. The complexity of performing (i), (ii), and (iii) is $O\left(N^{3}\right)$, $O\left(k N^{2}\right)$, and $O\left(N^{2} / k\right)$, respectively, where $k$ denotes the average number of variables detected in one iteration and $k \ll N$ for a typical threshold value. The complexity of performing (iv) is insignificant relative to the other three items because it merely involves problems with only two variables. 3) In addition to the basic algorithm, the complexity required by the 1-bit, 2-bit, and combined 1-bit-and-2-bit descent search is on the order of $O\left(N^{2}\right)$, which is insignificant relative to that of the basic algorithm.

\section{E. Extension to 64-QAM OFDM Systems}

With minor modifications, the proposed algorithm can readily be extended to 64-QAM OFDM systems. In this case, the ICI reduction problem can be formulated as

$$
\begin{aligned}
& \operatorname{minimize} \hat{\mathbf{X}}^{T} \hat{\mathbf{Q}} \hat{\mathbf{X}}+\hat{\mathbf{q}}^{T} \hat{\mathbf{X}} \\
& \text { subject to: } \hat{X}_{k}=\{ \pm 1, \pm 3, \pm 5, \pm 7\}, \\
& \text { for } k=0, \ldots, 2 N-1
\end{aligned}
$$

where $\hat{\mathbf{Q}}=\hat{\mathbf{A}}^{T} \hat{\mathbf{A}}, \hat{\mathbf{q}}=-2 \hat{\mathbf{A}}^{T} \hat{\mathbf{Y}}$. The variable set in (26b) can be characterized as

$$
\hat{\mathbf{X}}=4 \boldsymbol{\alpha}+2 \boldsymbol{\beta}+\boldsymbol{\gamma}
$$

where $\boldsymbol{\alpha}, \boldsymbol{\beta}$, and $\boldsymbol{\gamma}$ are $2 N$-dimensional vectors with components $\alpha_{k}, \beta_{k}$, and $\gamma_{k} \in\{-1,1\}$, for $k=0, \ldots, 2 N-1$. Problem (26) then assumes the form

$$
\begin{aligned}
\operatorname{minimize} & \mathbf{z}^{T} \mathbf{Q z}+\mathbf{q}^{T} \mathbf{z} \\
\text { subject to: } & z_{k}=\{-1,1\}, \text { for } k=0, \ldots, 6 N-1
\end{aligned}
$$

with $\mathbf{z}=\left[\begin{array}{l}\boldsymbol{\alpha} \\ \boldsymbol{\beta} \\ \gamma\end{array}\right], \mathbf{Q}=\left[\begin{array}{ccc}16 \hat{\mathbf{Q}} & 8 \hat{\mathbf{Q}} & 4 \hat{\mathbf{Q}} \\ 8 \hat{\mathbf{Q}} & 4 \hat{\mathbf{Q}} & 2 \hat{\mathbf{Q}} \\ 4 \hat{\mathbf{Q}} & 2 \hat{\mathbf{Q}} & \hat{\mathbf{Q}}\end{array}\right]$, and $\mathbf{q}=\left[\begin{array}{c}4 \hat{\mathbf{q}} \\ 2 \hat{\mathbf{q}} \\ \hat{\mathbf{q}}\end{array}\right]$. 
The relaxation and solution technique described in Sections 3 and 4.A-C can then be applied to problem (28) with straightforward modifications.

\section{Performance Evaluation}

The proposed algorithm was applied to an OFDM system where the number of subcarriers was set to $N=64$, and the length of the cyclic prefix was chosen to be $N_{p}=N / 8$. A twotap WSSUS fading channel was employed, where each channel tap is an independent complex Gaussian random process with Jakes' Doppler spectrum. The delay of the first tap was set to zero, and the delay of the second tap was randomly generated with uniform distribution from $\left\{T_{c}, \ldots, N_{p} T_{c}\right\}$. The normalized Doppler frequency of the channel is denoted as $f_{d} T_{s}$.

First, 16-QAM modulation was assumed in an OFDM system with a bandwidth of $200 \mathrm{kHz}$. Simulations were carried out to evaluate the performance of the proposed ICI reduction algorithm in OFDM systems based on bit error rate (BER) and computational complexity. The BER performance of the proposed algorithm was compared with that of the conventional one-tap equalizer and the DFE algorithm [8] under different system configurations. For the DFE algorithm, the number of neighbouring subcarriers used to suppress the ICI at a particular subcarrier was set to 25 .

64-QAM modulation was also employed in an OFDM system to demonstrate the performance of the proposed algorithm with various Doppler spreads. Perfect channel information was assumed, and combined 1-bit-and-2-bit LBDS was adopted to improve system performance.

The BER performance was evaluated in an OFDM system with $f_{d} T_{s}=0.05$. The BER versus signal-to-noise ratio (SNR) of the proposed ICI reduction algorithm is shown in Fig. 1. The performance of the one-tap equalizer and DFE algorithm are also plotted in the figure to compare the performance. It can be observed that the one-tap equalizer provides unsatisfactory performance in time-varying channels, but the proposed algorithm considerably mitigates intercarrier interference. The proposed algorithm outperforms the DFE algorithm with a slightly increased computational complexity. This is because the DFE algorithm only considers the ICI components of the $K-1$ neighboring subcarriers of the desired subcarrier, so the ICI components outside this range are discarded. However, the proposed algorithm utilizes all the ICI components to detect the transmitted signals. For example, at an SNR of $35 \mathrm{~dB}$, the DFE algorithm has a BER of $8 * 10^{-5}$, while the proposed algorithm with $\rho=0.8 \max \left|\mathbf{z}_{i}^{*}\right|$ has a BER of $5 * 10^{-5}$ (with a $20 \%$ increase in computational complexity). which can be further improved to a BER of $3.5 * 10^{-5}$ with LBDS (and a further $1 \%$ increase in computational complexity).

A larger threshold $\rho$ provides better performance at a cost of increased computational complexity, as shown in Figs. 1-3. The proposed algorithm exhibits an error floor at high SNR for a smaller threshold, but this can be effectively suppressed by performing LBDS with a slightly increased computational complexity. For a larger threshold, the system has comparable performance with and without LBDS, and the increase in computational complexity using LBDS is insignificant, i.e., for the OFDM system with Doppler spread $f_{d} T_{s}=0.1$, the algorithm with $\rho=0.5 \max \left|\mathbf{z}_{i}^{*}\right|$ requires $15 \%$ more CPU time than that with the DFE algorithm with a BER of 0.002 at an SNR of $35 \mathrm{~dB}$. This can be improved to $2 * 10^{-5}$ by performing LBDS with $20 \%$ more CPU time than the DFE algorithm. While the algorithm with $\rho=0.8 \max \left|\mathbf{z}_{i}^{*}\right|$ requires $25 \%$ more CPU time than the DFE algorithm, but achieves a BER of $4 * 10^{-5}$, which can be improved to $1.5 * 10^{-5}$ by performing LBDS with $26 \%$ more CPU time than the DFE algorithm.

Simulations were also carried out to determine the impact of normalized doppler spread $f_{d} T_{s}$ on the system performance. The BER performance of the proposed algorithm for $f_{d} T_{s}=$ 0.1 and $f_{d} T_{s}=0.3$ is depicted in Figs. 2 and 3, respectively. It can be observed that the system performance improves as the Doppler spread increases. For example, for $\rho=0.8 \max \left|\mathbf{z}_{i}^{*}\right|$, an SNR of $33 \mathrm{~dB}$ is required to achieve a BER of $10^{-4}$ for the case $f_{d} T_{s}=0.1$ with LBDS, while with $f_{d} T_{s}=0.3$, an SNR of $31.4 \mathrm{~dB}$ is required to achieve the same BER. This improvement with increasing $f_{d} T_{s}$ can be attributed to the increased temporal diversity introduced by the larger Doppler spread [15]. Similar diversity gain can also be achieved in 64QAM OFDM systems by employing the proposed algorithm, as shown in Fig. 4.

\section{CONCLUSIONS}

A new low complexity ICI reduction algorithm for OFDM systems with doubly-selective fading channels and QAM signal constellations has been proposed. A combinatorial optimization problem for ICI suppression was formulated, and then relaxed to a QP problem. A successive method was then utilized to deduce a sequence of reduced-size QP problems, which were solved by limiting the search in the 2-dimensional subspace spanned by its steepest-descent and Newton directions to reduce the computational complexity. Furthermore, a low-bit descent search was employed to improve the system performance. Several implementation efficiency issues were addressed. Results were given which demonstrated that the proposed algorithm provides excellent performance with low computational complexity.

\section{REFERENCES}

[1] R. V. Nee and R. Prasad, OFDM for Wireless Multimedia Communications, Artech House, 2000.

[2] ETSI, "Digital video broadcasting: framing structure, channel coding, and modulation for digital terrestrial television," European Telecommunication Standard, ETS 300-744, Aug. 1997.

[3] ETSI, "Radio broadcasting systems: digital audio broadcasting to mobile, portable and fixed receivers," European Telecommunication Standard, ETS 300-401, Feb. 1995.

[4] IEEE 802.11, IEEE Standard for Wireless LAN: Medium Access Control (MAC) and Physical Layer (PHY) specifications, Nov. 1997.

[5] IEEE 802.16, "IEEE Standard for Local and metropolitan area networks: Air Interface for Fixed Broadband Wireless Access Systems," June 2004. 
[6] Y. G. Li and L. J. Cimini, Jr., "Bounds on the interchannel interference of OFDM in time-varying impairments," IEEE Trans. Commun., vol. 49 no. 3, pp. 401-404, Mar. 2001.

[7] W.-S. Hou and B.-S. Chen, "ICI cancellation for OFDM communication systems in time-varying multipath fading channels," IEEE Trans. Wireless Commun., vol. 4, no. 5, pp. 2100-2110, Sept. 2005.

[8] X. Cai and G. B. Giannakis, "Bounding performance and suppressing intercarrier interference in wireless mobile OFDM," IEEE Trans. Commun., vol. 51, no. 12, pp. 2047-2056, Dec. 2003.

[9] Y. J. Kou, W.-S. Lu, and A. Antoniou, "An iterative intercarrierinterference reduction algorithm for OFDM systems," Proc. IEEE Pacific Rim Conf. on Commun., Computers, and Signal Process., pp. 538-541, Aug. 2005.

[10] A. Stamoulis, S. N. Diggavi, and N. Al-Dhahir, "Intercarrier interference in MIMO OFDM," IEEE Trans. Signal Process., vol. 50, no. 10, pp. 2451-2464, Oct. 2002.

[11] J. G. Proakis, Digital Communications, 4th Ed., McGraw-Hill, 2001.

[12] M. Russell and G. L. S. Stuber, "Interchannel interference analysis of OFDM in a mobile environment," Proc. IEEE Vehic. Tech. Conf., pp. 820-824, July 1995.

[13] R. A. Horn and C. R. Johnson, Matrix Analysis, Cambridge University Press, 1985.

[14] W.-S. Lu, "Design of FIR digital filters with discrete coefficients via convex programming," Proc. IEEE ISCAS, pp. 1831-1834, May 2005.

[15] N. J. Bass and D. P. Taylor, "Matched filter bounds for wireless communication over Rayleigh fading dispersive channel," IEEE Trans. Commun., vol. 49, no. 9, pp. 1525-1528, Sept. 2001.

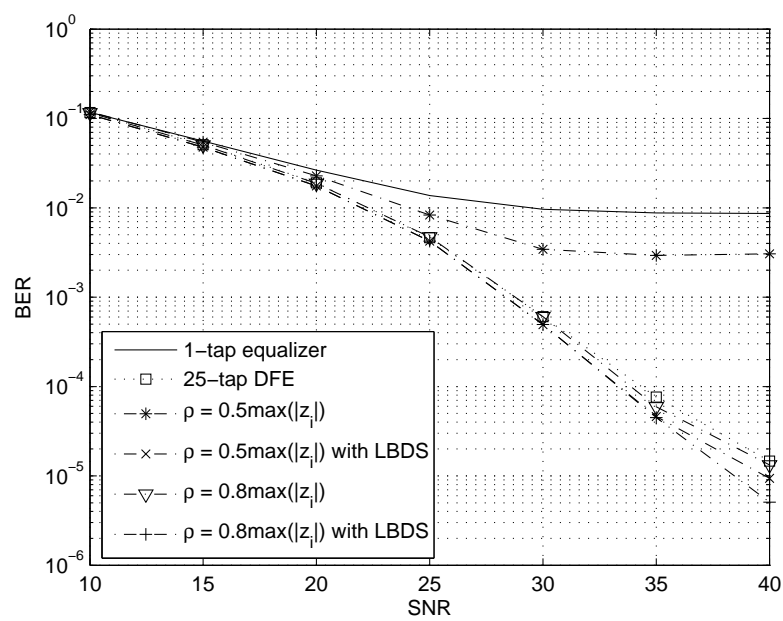

Fig. 1. BER performance with $f_{d} T_{s}=0.05$ in a 16-QAM OFDM system.

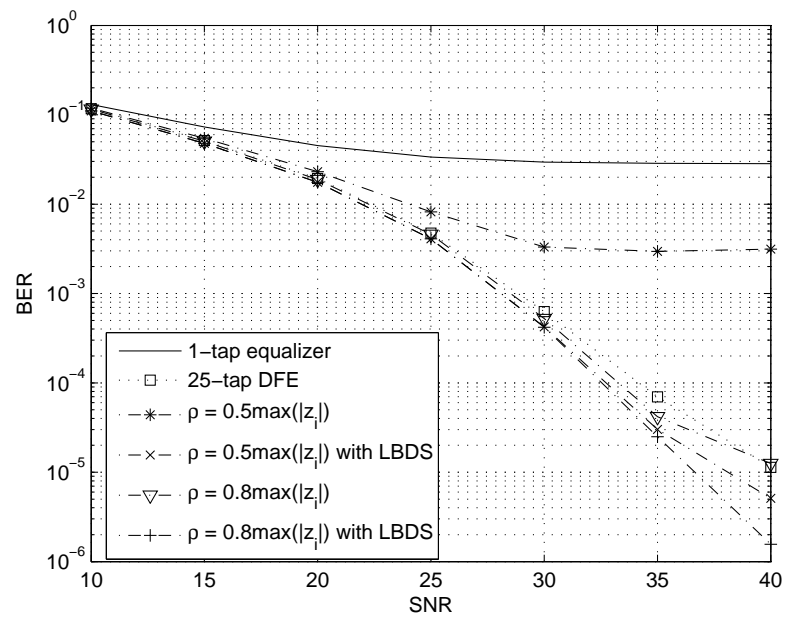

Fig. 2. BER performance with $f_{d} T_{s}=0.1$ in a 16-QAM OFDM system.

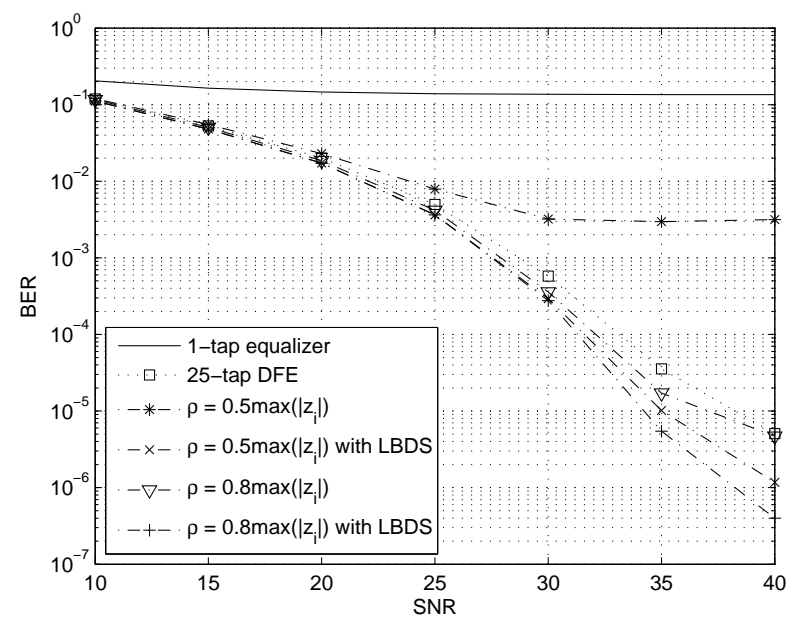

Fig. 3. BER performance with $f_{d} T_{s}=0.3$ in a 16-QAM OFDM system.

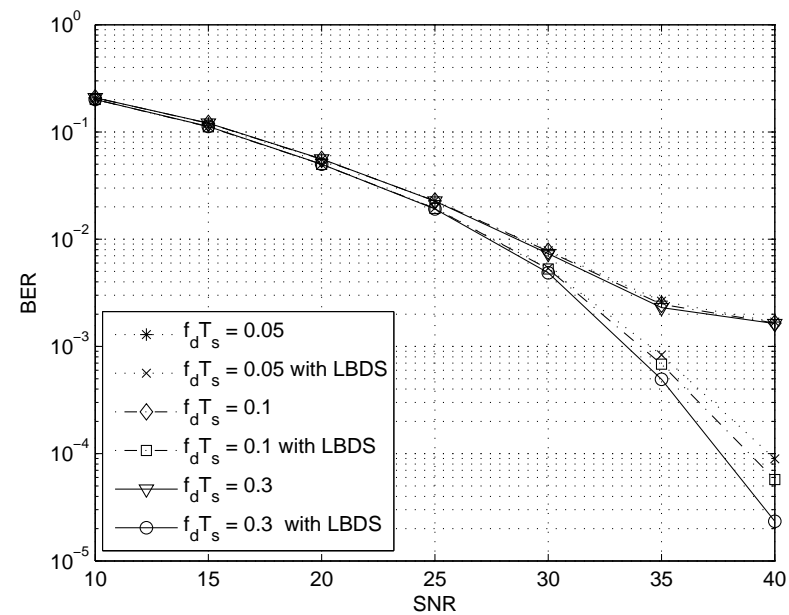

Fig. 4. BER performance with various Doppler spreads in a 64-QAM OFDM system. 